\title{
The demographic and clinical profiles of women presenting with vaginal discharge syndrome at primary care facilities in South Africa: Associations with age and implications for management
}

\author{
T Kufa ${ }^{1,2}$ MB ChB, PhD; L Gumede, ${ }^{1}$ Nat Dip Med Tech; D V Maseko, ${ }^{1}$ Nat Dip Med Tech, BTech; F Radebe, ${ }^{1}$ Nat Dip Med Tech, MSc; \\ R Kularatne, ${ }^{1,3}$ MB ChB, MSc (Med), FC Path (SA) Micro \\ ${ }^{1}$ Centre for HIV and STIs, National Institute for Communicable Diseases, Johannesburg, South Africa \\ ${ }^{2}$ School of Public Health, Faculty of Health Sciences, University of the Witwatersrand, Johannesburg, South Africa \\ ${ }^{3}$ Department of Clinical Microbiology and Infectious Diseases, Faculty of Health Sciences, University of the Witwatersrand, Johannesburg, \\ South Africa
}

Corresponding author: T Kufa (tendesayikc@nicd.ac.za)

\begin{abstract}
Background. Current South African guidelines for the management of vaginal discharge syndrome (VDS) do not recommend treatment for sexually transmitted infection (STI) pathogens for women aged $\geq 35$ years whose partners do not have male urethritis syndrome. The guideline assumes that older women are unlikely to have an STI and that their partners do not have asymptomatic infections.

Objectives. To describe the demographic, behavioural and clinical characteristics of women with VDS, comparing older women ( $\geq 35$ years) with younger women, and to determine the performance of age alone as a criterion for predicting the presence of STI.

Methods. This was a cross-sectional study at seven primary healthcare centres taking part in the aetiological surveillance of STIs between January 2015 and December 2016. Eligible women presenting with VDS were enrolled and completed a nurse-administered questionnaire. Genital swabs and blood specimens were collected for laboratory testing. Data were entered into surveillance-specific databases and exported into Stata 14 for analysis. Descriptive statistics were used to compare demographic and clinical profiles of older with younger women. A receiver operator curve (ROC) was used to determine the age cut-off that would best differentiate between women who had infection with STI pathogens and those without.

Results. Of 757 women enrolled, 157 (20.7\%) were aged $\geq 35$ years. HIV positivity was $46.6 \%$, and higher in older than younger women (54.9\% v. 44.5\%; $p=0.02$ ). Of those enrolled, $283(37.4 \%)$ had bacterial vaginosis (BV) and/or Candida infection only, 232 (30.7\%) had BV or Candida with STI pathogens detected, 98 (13\%) were infected with STI pathogens only, and 144 (19.0\%) did not have any detectable STI or non-STI causes. Although older women were less likely than younger women to have Neisseria gonorrhoeae, Chlamydia trachomatis or Mycoplasma genitalium infection ( $23.6 \%$ v. $38.2 \%$; $p<0.01)$, the burden in older women was not negligible. The area under the ROC for age was $57.5 \%$ (95\% confidence interval 53.2 - 61.8\%), which implies suboptimal performance.

Conclusions. Although older women with VDS were less likely than younger women to have STIs, a significant proportion of them did have an infection with STI pathogens. Age alone was not a good criterion for discriminating between women with and without infection with STI pathogens. Other ways of improving the VDS algorithm performance are needed, as is better integration of HIV and STI prevention and treatment.
\end{abstract}

S Afr Med J 2018;108(10):876-880. DOI:10.7196/SAMJ.2018.v108i10.13027

Since 1995, South Africa (SA) has used the syndromic management approach to manage sexually transmitted infections (STIs). ${ }^{[1]}$ This approach uses standardised flow charts and treatment algorithms to identify and treat individuals with similar symptoms and clinical signs that are associated with common aetiologies. ${ }^{[2,3]}$ Syndromic management was implemented in SA, as in other resource-limited settings, to address the limited laboratory capacity to diagnose the different causes before initiating appropriate treatment. ${ }^{[3]}$ The introduction of syndromic management allowed STI treatment to be provided in the same visit without the risk of loss to follow-up and ongoing transmission to partners, at a cost that was affordable to the country. However, syndromic management has its disadvantages, mainly suboptimal performance in predicting presence of STIs, particularly in women with vaginal discharge syndrome (VDS). This suboptimal performance has been documented in diverse populations of women, including women living with HIV, women at high risk of HIV acquisition, and women attending STI services who were either symptomatic or asymptomatic. ${ }^{[3-11]}$ When compared with the gold standard of laboratory diagnosis, syndromic management was found to have sensitivities (defined as the proportion of women with STIs who are classified as having VDS and therefore correctly treated) ranging from $5 \%$ to $100 \% \cdot{ }^{[3,11]}$ Sensitivities of VDS syndromic management algorithms were higher for vaginal STI infections (bacterial vaginosis (BV) or Trichomonas vaginalis) than for cervical infections (Neisseria gonorrhoeae, Chlamydia trachomatis or Mycoplasma genitalium). On the other hand, VDS algorithms also have poor positive predictive values - defined as the proportion of women with VDS who turn out to have an STI on laboratory diagnosis and giving a measure of the proportions who are overtreated according to the algorithm - ranging from $9 \%$ to $47.5 \% .^{[3,11]}$ This poor performance means that some women with STIs go untreated, as infections can be asymptomatic, while others are 
treated unnecessarily. Syndromic management algorithms are also not useful for STI infections at extragenital sites such as the pharynx or anus. ${ }^{[12]}$

In the 2014 - 2015 fiscal year, 1.1 million people were treated for at least one new STI syndrome in SA. ${ }^{[13]}$ VDS was common among STI clinic attendees, and seen in $42 \%$ of patients presenting with STI syndromes (National Institute for Communicable Diseases (NICD) sentinel surveillance data, unpublished). Current syndromic management guidelines for VDS recommend treatment with ceftriaxone, azithromycin and metronidazole for women aged $<35$ years or those who are older but have partners with symptoms of male urethritis syndrome (MUS). ${ }^{[14]}$ This treatment covers the most common treatable and sexually transmitted causes of vaginal discharge in women, namely N. gonorrhoeae, C. trachomatis, T. vaginalis and M. genitalium. For women aged $\geq 35$ years whose partners have no MUS, only metronidazole and clotrimazole therapy are recommended. This guideline assumes that these older women have BV or Candida infection only - traditionally considered to be non-STI causes of vaginal discharge. ${ }^{[14]}$ The guidelines also assume that these women's partners are not asymptomatic, although a significant proportion of at-risk males may have asymptomatic urethral STIs. ${ }^{[15]}$ In addition, the syndromic management guidelines recommend behavioural risk reduction counselling, HIV testing and linkage to care and treatment, condom promotion, demonstration and provision, conception and contraception counselling, and partner management.

\section{Objectives}

To describe the demographic, behavioural and clinical characteristics of women with VDS enrolled at primary healthcare centres (PHCs) in SA, comparing those aged $\geq 35$ years with those who were younger. We also assessed the performance of age as a criterion for excluding STIs and discuss the implications of the findings in terms of management of VDS in in the country.

\section{Methods \\ Study design}

This was a cross-sectional study of women aged $\geq 18$ years presenting with VDS to seven STI sentinel surveillance sites in five provinces.

\section{Setting}

The Centre for HIV and STIs at the NICD has conducted sentinel site-based aetiological surveillance of STIs since 2004. The objective of the surveillance is to monitor the aetiological causes of STI syndromes and gonococcal antimicrobial resistance in SA. This surveillance also validates the recommended treatment regimens and ensures that the treatments included cover the most common causative STI pathogens for each syndrome. During the period 1 January 2015 - 31 December 2016, sentinel surveillance was conducted at seven PHCs located in five provinces - Eastern Cape, Gauteng, KwaZulu-Natal, Mpumalanga and North West.

\section{Data collection}

Following completion of eligibility assessment and informed consent procedures, consecutive women aged $\geq 18$ years presenting with VDS to seven PHCs between January 2015 and December 2016 were enrolled. Demographic and clinical data were collected using a nurse-administered questionnaire. Demographic variables included in the questionnaire included age, gender and race, while behavioural variables included condom use at last sexual encounter and having sexual partners from outside the country or province. Clinical variables were syndrome(s) diagnosed on the day of enrolment, treatment of STI syndromes in the preceding 12 months, non- resolution of STI symptoms in the preceding 3 months, and referral from another STI treatment provider. Participants were asked to provide vaginal and endocervical swabs as well as venous blood specimens for laboratory testing. Surveillance questionnaires were linked to laboratory specimens through a barcode with a unique identification number. No other identifying information was collected.

\section{Laboratory procedures}

Specimens were transported to the STI reference laboratory at the NICD in Johannesburg. DNA was extracted from endocervical swabs using two automated DNA extractors (X-tractor Gene and QIAxtractor platforms; Qiagen, Germany). Swab-extracted DNA was tested using a validated in-house real-time multiplex polymerase chain reaction assay on the RotorGene platform (Qiagen) to detect the presence of the STI pathogens N. gonorrhoeae, C. trachomatis, T. vaginalis and M. genitalium. Gram-stained smears of vaginal swab specimens were assessed microscopically for presence of BV (Nugent scoring) and Candida species. HIV seropositivity was determined using two rapid immunochromatographic assays, Unigold (Trinity Biotech, Ireland) and Alere Determine (Alere Medical Co. Ltd, Japan).

\section{Data management and analysis}

Data were captured into a study-specific Microsoft Access 2010 database (Microsoft, USA) and exported into Stata 14 (StataCorp, USA) for analysis. Descriptive statistics was used to describe the women enrolled, comparing women aged $\geq 35$ years with those who were younger. The $\chi^{2}$ test and rank-sum tests were used to test for associations between age category and other categorical and numerical variables. A $p$-value $<0.05$ was considered statistically significant. Simple proportions were used to describe the prevalence of STI and non-STI pathogen groups: (i) BV or Candida only; (ii) BV or Candida with STI pathogens; (iii) STI pathogens only; and (iv) no pathogens detected. A receiver operator curve (ROC) of age as a diagnostic criterion (with threshold values at $18-19,20-21$, $22-23,24-25,26-28,29-31,32-34,35-37,38-40,41-43$, $44-46,47-49$ and $\geq 50$ years) was used to determine an age that would best predict the presence of the N. gonorrhoeae, C. trachomatis or M. genitalium infections and the area under the curve (AUC) determined. This AUC represents the probability of any age category selected at random correctly discriminating between women with any one or more of the three STI pathogens and those without. These three infections were chosen for use in the ROC analysis because in accordance with the existing VDS algorithm, they would be treated with ceftriaxone and azithromycin. These drugs would not be given to older women whose partners did not have MUS. The study had a planned sample size of 700 based on expected enrolment of 100 women per sentinel site. The sample size of 100 per site was considered sufficient to determine $N$. gonorrhoeae prevalence rates of between $12 \%$ and $22 \%$ assuming an alpha level of 0.05 , power of $80 \%$ and no clustering of $N$. gonorrhoeae prevalence rates by site.

\section{Ethical considerations}

The study was approved by the University of the Witwatersrand Human Research Ethics Committee (ref. no. M160667). Written informed consent was obtained from all eligible women prior to administration of the questionnaire and specimen collection. To protect the privacy of the participants, the study was anonymous and unlinked. This means that no identifying information or contact details were collected and all study materials (questionnaires and specimens) were identified and linked through a unique study number. As syndromic management of STIs is standard of care for 
Table 1. Demographic, behavioural and clinical profiles of women with VDS by age at enrolment

\begin{tabular}{|c|c|c|c|c|}
\hline Characteristic & All $(N=757)$ & $\begin{array}{l}\text { Age }<35 \text { years } \\
(N=600)\end{array}$ & $\begin{array}{l}\text { Age } \geq 35 \text { years } \\
(N=157)\end{array}$ & $p$-value \\
\hline Enrolment at rural site, $n(\%)$ & $77(10.2)$ & $55(9.2)$ & $22(14.0)$ & 0.074 \\
\hline Age at first sex (years), median (IQR) & $18(16-19)$ & $18(16-19)$ & $18(16-20)$ & 0.927 \\
\hline Condom use at last sexual encounter, $n(\%)$ & $156(20.1)$ & $114(19.0)$ & $42(26.8)$ & 0.033 \\
\hline Treated for an STI syndrome in the past 12 months, $n$ (\%) & $243(32.1)$ & $186(31.0)$ & $57(36.3)$ & 0.205 \\
\hline $\begin{array}{l}\text { Non-resolution of symptoms or referral from another provider in the } \\
\text { preceding } 3 \text { months, } n(\%)\end{array}$ & $68(9.0)$ & $55(9.2)$ & $13(8.3)$ & 0.729 \\
\hline Concomitant genital ulcer disease at enrolment, $n(\%)$ & $62(8.1)$ & $54(9.0)$ & $8(5.1)$ & 0.112 \\
\hline Pathogen group & & & & 0.013 \\
\hline BV or Candida only & $283(37.4)$ & $227(37.8)$ & $56(35.7)$ & \\
\hline BV or Candida with an STI & $232(30.6)$ & $195(32.5)$ & $37(23.6)$ & \\
\hline STI only & $98(13.0)$ & $77(12.8)$ & $21(13.4)$ & \\
\hline No pathogen & $144(19.0)$ & $101(16.8)$ & $43(27.4)$ & \\
\hline Neisseria gonorrhoeae, $n(\%)$ & $140(18.5)$ & $121(20.2)$ & $19(12.1)$ & 0.02 \\
\hline Chlamydia trachomatis, $n(\%)$ & $134(17.7)$ & $118(19.7)$ & $16(10.2)$ & 0.006 \\
\hline Mycoplasma genitalium, $n$ (\%) & $65(8.6)$ & $59(9.8)$ & $6(3.8)$ & 0.017 \\
\hline N. gonorrhoeae OR C. trachomatis OR M. genitalium, $n(\%)$ & $266(35.1)$ & $229(38.2)$ & $37(23.6)$ & $<0.001$ \\
\hline Trichomonas vaginalis, $n(\%)$ & $108(14.3)$ & $78(13.0)$ & $30(19.1)$ & 0.051 \\
\hline $\mathrm{BV}, n(\%)$ & $427(56.4)$ & $348(58.0)$ & $79(50.3)$ & 0.084 \\
\hline Candida, $n(\%)$ & $167(22.1)$ & $148(24.8)$ & $19(12.1)$ & $<0.001$ \\
\hline HIV-positive, $n(\%)$ & $353(46.6)$ & $267(44.5)$ & $86(54.9)$ & 0.022 \\
\hline
\end{tabular}

VDS in SA, the laboratory results were not used for management and were not returned to participating women.

\section{Results \\ Description of the study population}

A total of 757 women were enrolled during the study period. The median age was 27 years (interquartile range (IQR) 22 - 32), $157(20.7 \%)$ were aged $\geq 35$ years, and $750(99.1 \%)$ were black Africans. Table 1 describes the demographic, behavioural and clinical characteristics of women by age category. Compared with the younger women, older women were more likely to report condom use at their last sexual encounter, but they were not different to the younger ones in respect of other behavioural factors or previous history of VDS. The overall HIV positivity was $46.6 \%$, with older women more likely than younger women to be HIV-seropositive (54.9\% v. $44.5 \%$; $p=0.022)$.

\section{Prevalence of non-STI and STI causes of VDS by age}

On laboratory testing, 283 women (37.4\%) had BV and/or Candida infection without an STI; 232 (30.7\%) had BV or Candida with at least one STI pathogen detected; 98 (12.9\%) had one or more STI pathogens detected but without BV or Candida; while 144 (19.0\%) did not have any detectable STI or non-STI causes. Compared with younger women ( $<35$ years), older women were less likely to have BV and/or Candida with a concomitant STI $(23.6 \% \mathrm{v}$. $32.5 \%$; $p=0.013$ ) but more likely to have no identifiable VDS aetiology $(27.4 \%$ v. $16.8 \%$; $p=0.013)$. Although older women were significantly less likely than younger women to have N. gonorrhoeae, C. trachomatis or M. genitalium infection ( $23.6 \%$ v. $38.2 \%$; $p<0.001)$, the proportions of older women with these STI pathogens detected on an endocervical swab were $12.1 \%, 10.2 \%$ and $3.8 \%$, respectively. Older women were also less likely than younger women to have Candida infection $(12.1 \%$ v. $24.8 \%$; $p<0.001)$, but just as likely to have BV $(50.3 \%$ v. $58.0 \% ; p=0.084)$ or $T$. vaginalis infection $(19.1 \%$ v. $13.0 ; p=0.051)$.

\section{Performance of age as a criterion for predicting} presence of STI pathogens

As age $\geq 35$ years was significantly associated with lower (but not negligible) rates of STI infections in women presenting with VDS, an ROC was plotted to determine the age that best differentiated women with STI pathogens (N. gonorrhoeae, C. trachomatis or M. genitalium) from those without (Fig. 1 and Table 2). The curve for age was close to the chance level with no clear-cut age threshold located in the ROC space. The AUC was 57.5\% (95\% confidence interval 53.2 - 61.8), which implied suboptimal performance.

\section{Discussion}

The objectives of this study were to compare demographic, behavioural and clinical characteristics of older women ( $\geq 35$ years) with VDS with those of younger women ( $<35$ years) and to determine an age threshold that best differentiated between women with

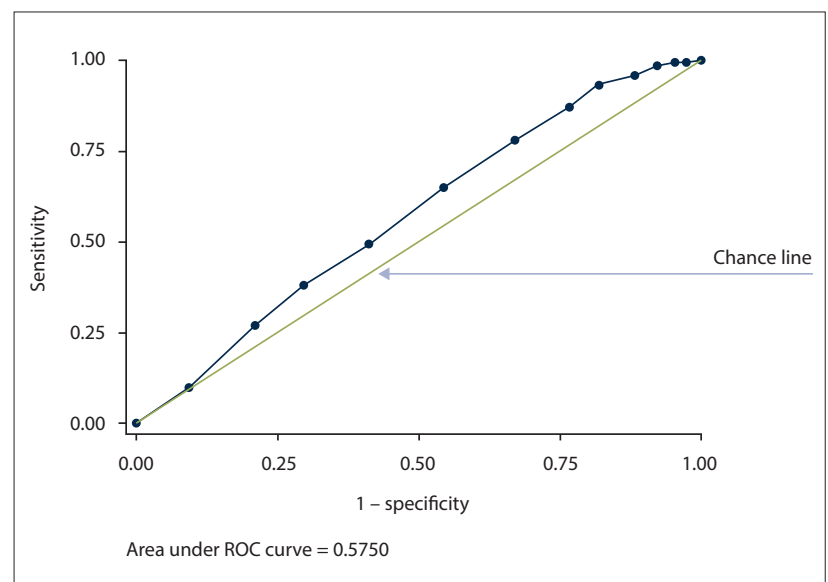

Fig. 1. ROC for performance of age in predicting presence of Neisseria gonorrhoeae, Chlamydia trachomatis or Mycoplasma genitalium. (ROC = receiver operator curve.) 


\begin{tabular}{|c|c|c|c|}
\hline Age category (years) & $\begin{array}{l}\text { Sensitivity for Neisseria gonorrhoeae, } \\
\text { Chlamydia trachomatis or Mycoplasma } \\
\text { genitalium, \% }\end{array}$ & $\begin{array}{l}\text { Specificity for N. gonorrhoeae, } \\
\text { C. trachomatis or M. genitalium, \% }\end{array}$ & Correctly classified, \% \\
\hline $18-19$ & 10.7 & 91.0 & 62.7 \\
\hline $20-21$ & 28.1 & 80.1 & 61.9 \\
\hline $22-23$ & 39.2 & 71.7 & 60.3 \\
\hline $24-25$ & 51.0 & 60.6 & 57.1 \\
\hline $26-28$ & 65.9 & 46.9 & 53.5 \\
\hline $29-31$ & 78.0 & 33.8 & 49.3 \\
\hline $32-34$ & 87.1 & 24.2 & 46.2 \\
\hline $35-37$ & 93.2 & 19.1 & 45.0 \\
\hline $38-40$ & 95.8 & 12.5 & 41.7 \\
\hline $41-43$ & 98.1 & 8.2 & 39.7 \\
\hline $44-46$ & 99.6 & 5.3 & 38.4 \\
\hline $47-49$ & 99.6 & 3.1 & 36.9 \\
\hline$\geq 50$ & 100 & 0.2 & 35.2 \\
\hline
\end{tabular}

treatable STI pathogens and those without. We found that although older women were significantly less likely than younger women to be infected with STI pathogens, the proportions of older women with detectable STI pathogens were not negligible. Moreover, there was no optimal age threshold that accurately differentiated between women with infection by treatable STI pathogens and those without.

These findings highlight a challenge that STI programmes in resource-limited settings face in improving the performance of VDS algorithms. Efforts to improve algorithm performance have sought to include other factors that may better distinguish the subset of VDS-positive women who have STIs from those with no STIs. Factors that have been evaluated include risk assessments based on self-reported high-risk sexual behaviour or a sexual history such as having a new sexual partner, having more than one sexual partner or having a symptomatic partner. ${ }^{\left[{ }^{[]}\right.}$Other criteria have included vaginal examination, vaginal microscopy or point-of-care (POC) testing. ${ }^{[3]}$ POC tests for N. gonorrhoeae or C. trachomatis alone or in combination conducted on urine, vaginal or endocervical swabs collected from symptomatic or asymptomatic women have been found to have sensitivities ranging from $40 \%$ to $99 \%$ with positive predictive values of $31-96 \% \cdot{ }^{[10,16]}$ Research on cost-effectiveness and on how best to incorporate the POC testing into current VDS algorithms is needed.

The very high HIV positivity among both younger and older women with VDS was concerning, although not unexpected. STIs are known to facilitate HIV acquisition, while HIV is known to alter the natural history of some STIs with tendencies towards increased severity, recurrence and poorer response to treatment. ${ }^{[17]}$ The integration of STI and HIV prevention and treatment needs to be strengthened to ensure that all HIV-positive women with VDS and their partners are tested for HIV and those who are HIV-positive are linked into care, while those who are HIV-negative access risk reduction counselling and are provided with condoms and if eligible referred for pre-exposure prophylaxis for HIV. A recent national assessment of STI services using standardised patient actors showed that $61 \%$ of STI patients were offered HIV testing, $30 \%$ were provided with condoms and $6 \%$ of males were offered male circumcision, with women less likely than men to be offered relevant services. ${ }^{[18]}$ With the development, introduction and scale-up of biomedical interventions such as microbicides, women with VDS should be a priority population for access.

\section{Study limitations}

Our analysis, which was intended to evaluate age as a criterion for improving the VDS algorithm, had some important limitations. This was a secondary analysis of sentinel surveillance data. As such, the variable needed to evaluate the full algorithm - such as whether or not the partner is symptomatic - was not available because it was not included in the surveillance questionnaire. Other variables such as knowledge of HIV status, ART use and viral suppression were not available, although they are important when evaluating populations with high HIV positivity such as ours.

\section{Conclusions}

Despite the above limitations, our study demonstrated that although older women with VDS were less likely to have STIs compared with younger women, a significant proportion of them did have STIs, and that age alone was not a good criterion for discriminating between women with and without infection with STI pathogens. Evaluation of the role of POC tests in improving the VDS algorithm performance is recommended, as is better integration of HIV and STI prevention and treatment.

\section{Acknowledgements. None.}

Author contributions. TK analysed and interpreted the data, and drafted and critically reviewed the manuscript; LG collected some data, interpreted the data and critically reviewed the manuscript; FR and DVM designed the study, interpreted the data and critically reviewed the manuscript; and RK interpreted the data and critically reviewed the manuscript. All authors approved the manuscript for submission.

Funding. The study was funded by the SA National Department of Health through a core surveillance funding grant to the NICD.

Conflicts of interest. None.

1. White RG, Moodley P, McGrath N, et al. Low effectiveness of syndromic treatment services for curable sexually transmitted infections in rural South Africa. Sex Transm Infect 2008;84(7):528-534. https:// doi.org/10.1136/sti.2008.032011

2. Henn E, Kruger T, Siebert T. Vaginal discharge reviewed: The adult pre-menopausal female. S Afr Fam Pract 2005;47(2):30-38. https://doi.org/10.1080/20786204.2005.10873183

Van Gemert C, Hellard M, Bradshaw CS, et al. Syndromic management of sexually transmissible infections in resource-poor settings: A systematic review with meta-analysis of the abnormal vaginal infections in resource-poor settings: A systematic review with meta-analysis of the abnormal vaginal discharge flowchart for Neisseria g

4. Aggarwal P, Bhattar S, Sahani SK, Bhalla P. Utility of laboratory diagnosis for confirmation of the Aggarwal P, Bhattar S, Sahani SK, Bhalla P. Utility of laboratory diagnosis for confirmation of the
syndromic case management in married Indian women with vaginal discharge. Int J Health Sci 2016:10(4):516-521. 
5. Chauhan V, Shah M, Thakkar S, Patel SV, Marfatia Y. Sexually transmitted infections in women: A correlation of clinical and laboratory diagnosis in cases of vaginal discharge syndrome. Indian Dermatol Online J 2014;5(Suppl 1):S1-S5. https://doi.org/10.4103/2229-5178.144498

6. Cornier N, Petrova E, Cavailler P, et al. Optimising the management of vaginal discharge syndrome in Bulgaria: Cost effectiveness of four clinical algorithms with risk assessment. Sex Transm Infect 2010;86(4):303-309. https:///doi.org/10.1136/sti.2009.040832

7. Djomand G, Gao H, Singa B, et al. Genital infections and syndromic diagnosis among HIVinfected women in HIV care programmes in Kenya. Int J STD AIDS 2016;27(1):19-24. https://doi. infected women in HIV care $\mathrm{p} 2$

8. Vishwanath S, Talwar V, Prasad R, Coyaji K, Elias CJ, de Zoysa I. Syndromic management of vaginal Vishwanath S, Talwar V, Prasad R, Coyaji K, Elias CJ, de Zoysa I. Syndromic management of vaginal
discharge among women in a reproductive health clinic in India. Sex Transm Infect 2000;76(4):303discharge among women in a reproductive

9. Van der Eem L, Dubbink JH, Struthers HE, et al. Evaluation of syndromic management guidelines for treatment of sexually transmitted infections in South African women. Trop Med Int Health 2016;21(9):1138-1146. https://doi.org/10.1111/tmi.12742

10. Nwankwo TO, Aniebue UU, Umeh UA. Syndromic diagnosis in evaluation of women with symptoms of vaginitis. Curr Infect Dis Rep 2017;19(1):3. https://doi.org/10.1007/s1 1908-017-0558-9

11. Zemouri C, Wi TE, Kiarie J, et al. The performance of the vaginal discharge syndromic management in treating vaginal and cervical infection: A systematic review and meta-analysis. PLoS One 2016;11(10):e0163365. https://doi.org/10.1371/journal.pone.0163365

12. Peters RP, Dubbink JH, van der Eem L, et al. Cross-sectional study of genital, rectal, and pharyngeal Chlamydia and gonorrhea in women in rural South Africa. Sex Transm Dis 2014;41(9):564-569. https://doi.org/10.1097/OLQ.0000000000000175
13. South African National AIDS Council. Enhanced Progress Report of the National Strategic Plan on HIV, STIs and TB: 2012 - 2016. SANAC, March 2016. http://sanac.org.za/wp-content/uploads/2016/07/ EPR-SANAC-NEW-DOC-latest-update3.pdf (accessed 23 August 2018).

14. National Department of Health, South Africa. Sexually Transmitted Infections: Management Guidelines 2015. https://www.idealclinic.org.za/docs/National-Priority-Health-Conditions/Sexually\%20Transmitted \%20Infections_\%20Management\%20Guidelines\%202015.pdf (accessed 23 August 2018).

15. Lewis DA, Chirwa TF, Msimang VM, Radebe FM, Kamb ML, Firnhaber CS. Urethritis/cervicitis Lewis DA, Chirwa TF, Msimang VM, Radebe FM, Kamb ML, Firnhaber CS. Urethritis/cervicitis pathogen prevalence and associated risk factors among asymptomatic HIV-infected patients in
Africa. Sex Transm Dis 2012;39(7):531-536. https://doi.org/10.1097/OLQ.0b013e31824cbecc

16. Herbst de Cortina S, Bristow CC, Davey DJ, Klausner JD. A systematic review of point of care testing for Chlamydia trachomatis, Neisseria gonorrhoeae, and Trichomonas vaginalis. Infect Dis Obste Gynecol 2016;2016:4386127. https://doi.org/10.1155/2016/4386127

17. Moodley P, Wilkinson D, Connolly C, Sturm AW. Influence of HIV-1 coinfection on effective management of abnormal vaginal discharge. Sex Transm Dis 2003;30(1):1-5. https://journals lww.com/stdjournal/Fulltext/2003/01000/Influence_of_HIV_1_Coinfection_on_Effective.1.aspx (accessed 4 September 2018).

18. Kohler PK, Marumo E, Jed SL, et al. A national evaluation using standardised patient actors to assess STI services in public sector clinical sentinel surveillance facilities in South Africa. Sex Transm Infect 2017;93(4):247-252. https://doi.org/10.1136/sextrans-2016-052930

Accepted 12 April 2018 